\title{
Characteristics of the Kepler target stars
}

\author{
Natalie M. Batalha ${ }^{1}$, William J. Borucki ${ }^{2}$, David G. Koch ${ }^{2}$ \\ Timothy M. Brown ${ }^{3}$, Douglas A. Caldwell ${ }^{4}$ and David W. Latham ${ }^{5}$ \\ ${ }^{1}$ Department of Physics and Astronomy, San Jose State University, San Jose, CA 95192 \\ ${ }^{2}$ Mailstop 244-30, NASA Ames Research Center, Moffett Field, CA 94035 \\ ${ }^{3}$ Las Cumbres Observatory Global Telescope Network, 6740 Cortona Dr., Ste 102, Goleta, CA \\ ${ }^{4}$ SETI Institute, 515 N. Whisman Rd., Mountain View, CA 94043 \\ ${ }^{5}$ Harvard-Smithsonian Center for Astrophysics, 60 Garden St., Cambridge, MA 02138
}

\begin{abstract}
The Kepler Mission successfully launched March 6, 2009, beginning its 3.5-year mission to determine the frequency of Earth-size planets in the habitable zones of late-type stars. The brightnesses of over 100,000 stars are currently being monitored for transit events with an expected differential photometric precision of $20 \mathrm{ppm}$ at $\mathrm{V}=12$ for a 6.5 -hour transit. The same targets will be observed continuously over the mission duration in order to broaden the detection space to orbital periods comparable to that of Earth. This paper provides an overview of the selection and prioritization criteria used to choose the stars that Kepler is observing from the $>4.5$ million objects in the 100 square degree field of view. The characteristics of the Kepler targets are described as well as the implications for detectability of planets in the habitable zone smaller than $2 \mathrm{R}_{\oplus}$.
\end{abstract}

A ground-based observing campaign was initiated several years before launch to determine the apparent magnitude (Kepler passband), the surface gravity, effective temperature, and stellar radius of all stars in the Kepler field using multi-color (g,r,i,z, and D51) photometry (Borucki et al. 2008). This effort required multiple visits to more than 1,600 pointings. The multi-color photometry was fit to synthetic Castelli \& Kurucz (2004) models (transformed to agree with observed M67 star colors) and constrained by a Bayesian prior probability distribution based on the statistics of stars in the solar neighborhood. Completeness with regards to stellar classification is at the $80 \%$ level down to 17 th magnitude. The resulting stellar properties are archived in the Kepler Input Catalog which is publicly available at the Multi-Mission Archive at Space Telescope Science Institute. The derived parameters form the basis of target selection and prioritization.

Given effective temperature, surface gravity, stellar radius, expected photometric precision, and a measure of the crowding in the photometric aperture, we compute the minimum detectable planet radius, $R_{p, \min }$, for every star at 1 ) the inner edge of the habitable zone; 2 ) half that distance; and 3) an orbit 5 stellar radii from the central star. The crowding metric is derived from simulated images using data from the Kepler Input Catalog and knowledge of the instrument characteristics (e.g. random noise sources, pixel response function, etc). The crowding is used to determine the dilution of the transit which translates to a larger $R_{p, \min }$. The end product is a sample of $\sim 188,000$ stars brighter than 16th magnitude that fall on silicon for which a planet of radius $\leqslant 2 R_{\oplus}$ is detectable (at acceptable confidence levels) over the duration of the mission. We note that stellar variability is not simulated for the purposes of target selection.

The target list is prioritized according to a weighted combination of $R_{p, \text { min }}$, apparent magnitude, and habitability (planets in the $\mathrm{HZ}$ are given higher priority). The list is then trimmed to meet flight segment constraints $(\leqslant 170,000$ targets and $\leqslant 5.44$ million pixels). 


\begin{tabular}{crrrrrrrrr}
\hline $\mathrm{Mag} / \mathrm{T}_{\mathrm{e}}(\mathrm{K})$ & 10500 & 9500 & 8500 & 7500 & 6500 & 5500 & 4500 & 3500 & Total \\
\hline 7.5 & 2 & 8 & 8 & 8 & 8 & 7 & 0 & 0 & 41 \\
8.5 & 8 & 20 & 26 & 24 & 50 & 16 & 7 & 8 & 159 \\
9.5 & 9 & 31 & 81 & 65 & 117 & 88 & 11 & 4 & 406 \\
10.5 & 27 & 37 & 100 & 209 & 405 & 362 & 40 & 9 & 1189 \\
11.5 & 24 & 58 & 172 & 396 & 1495 & 1356 & 157 & 39 & 3697 \\
12.5 & 33 & 43 & 230 & 678 & 4148 & 4761 & 625 & 62 & 10580 \\
13.5 & 34 & 51 & 170 & 737 & 9250 & 15841 & 2218 & 159 & 28460 \\
14.5 & 3 & 0 & 0 & 0 & 4791 & 29291 & 4401 & 552 & 39038 \\
15.5 & 7 & 3 & 0 & 0 & 4261 & 43132 & 11188 & 1828 & 60419 \\
\hline Total & 147 & 251 & 787 & 2117 & 24525 & 94854 & 18647 & 2661 & 143989 \\
\hline
\end{tabular}

The above Table reports the star counts (stars with $\log g \geqslant 3.5$ from amongst the 160,000 highest priority targets), binned by apparent magnitude and effective temperature. 16,011 stars $(\log g<3.5)$ are not represented in Table 1 . The smaller giants make it into the target sample when the apparent magnitude is bright enough to allow for the detection of planets as small as $2 \mathrm{R}_{\oplus}$ in an orbit as close as $5 \mathrm{R}_{*}$. The targets are dominated by G-type stars on or near the Main Sequence and stars fainter than 14th magnitude. At the temperature extremes, we have $~ 3,000$ M-type Main Sequence stars (low intrinsic brightness) and $<200 \mathrm{O}$ and B-type stars. OB-type stars are rare due not only to their short MS lifetimes, but also due to the fact that the field of view was chosen to avoid young stellar populations (OB associations and star forming regions).

We compute the number of detections expected given the target star population described above assuming that every star has a terrestrial-sized planet in its habitable zone. The calculation includes a red noise contribution derived from the solar irradiance variations. It yields an expected detection rate of hundreds of planets with radii $\leqslant 2 \mathrm{R}_{\oplus}$ in the habitable zone. A cursory look at the first 30 days of science data of the 20 brightest $G$ dwarfs in the sample suggests that stars as photometrically quiet as the Sun are as common as was expected by examining galactic populations models, the age-rotation-activity relation, and standard spin-down rates (Batalha et al. 2002). In particular, we identify 6 of the 20 that are indistinguishable (in terms of the stellar variability characteristics) from SOHO irradiance data of similar duration. The pre-selection of targets, made possible by pre-launch classification of the stars in the field of view, will significantly decrease the number of false positives so that the ground-based follow-up resources can be used more efficiently.

\section{Acknowledgements}

Kepler was selected as the 10th mission of the Discovery Program. Funding for this mission is provided by NASA, Science Mission Directorate.

\section{References}

Batalha, N. M., Jenkins, J., Basri, G. S., Borucki, W. J., \& Koch, D. G. 2002, in: F. Favata, I.W. Roxburgh \& D. Galadi (eds.), Stellar Structure and Habitable Planet Finding, ESA SP-485, p. 35

Borucki, W., Koch, D., Basri, G., Batalha, N., Brown, T., Caldwell, D., Christensen-Dalsgaard, J., Cochran, W., Dunham, E., Gautier, T. N., Geary, J., Gilliland, R., Jenkins, J., Kondo, Y., Latham, D., Lissauer, J. J., \& Monet, D. 2008, in: Y.-S. Sun, S. Ferraz Mello \& J.-L. Zhou (eds.), Exoplanets: Detection, Formation, and Dynamics, Proc. of IAU Symposium No 249 (Cambridge: CUP), p. 17

Castelli, F. \& Kurucz, R. L. 2004, arXiv:astro-ph/0405087 